Service social

\title{
Qui sont les jeunes adultes adoptant des conduites sursexualisées? Profil sociodémographique et difficultés sexuelles
}

\author{
Audrey Brassard, Élise Lachapelle, Mélanie Bourassa et Catherine De \\ Pierrepont
}

Volume 64, numéro 1, 2018

URI : https://id.erudit.org/iderudit/1055888ar

DOI : https://doi.org/10.7202/1055888ar

Aller au sommaire du numéro

Éditeur(s)

École de service social de l’Université Laval

ISSN

1708-1734 (numérique)

Découvrir la revue

Citer cet article

Brassard, A., Lachapelle, É., Bourassa, M. \& De Pierrepont, C. (2018). Qui sont les jeunes adultes adoptant des conduites sursexualisées? Profil

sociodémographique et difficultés sexuelles. Service social, 64(1), 15-29.

https://doi.org/10.7202/1055888ar

\section{Résumé de l'article}

Plusieurs chercheurs s'inquiètent de la sexualisation de l'espace public et de ses répercussions potentielles sur les adolescents et les adultes. Or, peu d'études documentent la sursexualisation des jeunes adultes, soit l'intégration des messages sexuels par l'adoption de conduites de surinvestissement de l'apparence, d'objectification, de séduction, d'un discours sexualisé, d'une sexualité axée sur la performance et d'un faible sens intime accordé à la sexualité (Brassard et al., 2016). Cette étude explore les caractéristiques sociodémographiques et les difficultés sexuelles des jeunes adultes adoptant des conduites sursexualisées. Les résultats de l'étude en ligne menée auprès de 1275 adultes de 18 à 30 ans montrent que les conduites sursexualisées varient selon le genre, l'âge, le statut conjugal, le genre du partenaire, la scolarité et l'expérience d'agression sexuelle dans l'enfance. Des liens nuancés émergent entre les conduites de sursexualisation et les difficultés sexuelles.
Tous droits réservés (C) Service social,
Ce document est protégé par la loi sur le droit d'auteur. L'utilisation des services d’Érudit (y compris la reproduction) est assujettie à sa politique d'utilisation que vous pouvez consulter en ligne.

https://apropos.erudit.org/fr/usagers/politique-dutilisation/ 


\title{
Qui sont les jeunes adultes adoptant des conduites sursexualisées? Profil sociodémographique et difficultés sexuelles
}

\author{
BRASSARD, Audrey, Ph.D. en psychologie, psychologue \\ Professeure au Département de psychologie
} Université de Sherbrooke

LACHAPELLE, Élise, bachelière et candidate au D.Ps. en psychologie Département de psychologie Université de Sherbrooke

BOURASSA, Mélanie, D.Ps. en psychologie, psychologue Université de Sherbrooke

DE PIERREPONT, Catherine, M.A. en sexologie, Ph.D. en santé des populations Stagiaire postdoctorale au Département de psychologie Université de Sherbrooke

\section{RÉSUMÉ}

Plusieurs chercheurs s'inquiètent de la sexualisation de l'espace public et de ses répercussions potentielles sur les adolescents et les adultes. Or, peu d'études documentent la sursexualisation des jeunes adultes, soit l'intégration des messages sexuels par l'adoption de conduites de surinvestissement de l'apparence, d'objectification, de séduction, d'un discours sexualisé, d'une sexualité axée sur la performance et d'un faible sens intime accordé à la sexualité (Brassard et al., 2016). Cette étude explore les caractéristiques sociodémographiques et les difficultés sexuelles des jeunes adultes adoptant des conduites sursexualisées. Les résultats de l'étude en ligne menée auprès de 1275 adultes de 18 à 30 ans montrent que les conduites sursexualisées varient selon le genre, l'âge, le statut conjugal, le genre du partenaire, la scolarité et l'expérience d'agression sexuelle dans l'enfance. Des liens nuancés émergent entre les conduites de sursexualisation et les difficultés sexuelles.

Mots-clés : jeunes adultes, sexualité, sursexualisation, difficultés sexuelles, profil.

Abstract

Many researchers worry about the sexualisation of the public space, and its repercussion on adolescents and adults. Yet, few studies have examined young adults' levels of oversexualisation, which is the integration of sexual messages through overinvolment in one's sexualized appearance, sexual self-objectification, sexualized language, seduction, sexuality based on performance, and low meaningfulness of sexuality (Brassard et al., 2016). This study explores the sociodemographic characteristics and sexual difficulties of young adults endorsing oversexualized behaviors. Results of this online study conducted among 1275 young adults aged 18 to 30 show that oversexualized behaviors vary across gender, age, marital status, partner's gender, education, and childhood sexual abuse. Nuanced associations are found between oversexualized dimensions and sexual difficulties.

Keywords: young adults, sexuality, oversexualisation, sexual difficulties, profile. 


\section{INTRODUCTION}

Depuis plusieurs années, le concept d'hypersexualisation des jeunes a suscité l'intérêt du public et des chercheurs (p. ex., APA, 2007 ; Duquet et Quiénart, 2009 ; Nalkur, Jamieson et Romer, 2010). L'hypersexualisation se définit tantôt comme des conduites excessives de séduction chez les jeunes (Richard-Bessette, 2006) ou comme une combinaison de pratiques sexualisées (p. ex., jeux sexuels, cyberpornographie), de situations ou d'attitudes sexualisées (p. ex., souci de performance) chez les adolescents (Duquet et Quiénart, 2009). On associe son émergence à l'augmentation du contenu sexualisé dans les médias et l'Internet, à l'omniprésence de standards de beauté idéalisés et érotisés ainsi qu'à la contamination de la culture populaire par les stéréotypes pornographiques (Nalkur et al., 2010 ; Poulin, 2008 ; Wright, 2009). Or, des auteurs ont souligné l'importance de ne pas généraliser ce phénomène à l'ensemble des jeunes et ont à cet effet documenté que la sexualité précoce et l'adoption de pratiques sexuelles marginales n'étaient pas le lot de tous les jeunes Canadiens (Blais et al., 2009). La vaste étude PIXEL sur la santé sexuelle de 3389 jeunes Québécois de 17 à 29 ans (Lambert et al., 2017) documente dans le même sens la faible prévalence de la sexualité dite précoce (relation sexuelle vaginale avant l'âge de 14 ans) des hommes (5,8\%) et des femmes $(6,0 \%)$, ainsi que les faibles taux annuels, chez les jeunes actifs sexuellement, des pratiques dites marginales, telles que la sexualité de groupe ( $4,7 \%$ des jeunes) et les activités sociales sexualisées (hommes : $12,4 \%$; femmes : $8,2 \%$ ).

Selon la théorie de la "cultivation» (cultivation theory), les contacts récurrents avec les messages normalisés dans les médias entrainent des attitudes et des croyances similaires chez ceux qui les reçoivent (Gerbner et al., 1994). Ainsi, une plus grande exposition aux modèles et messages porteurs d'une objectification sexuelle des femmes mènerait à un risque plus grand d'identification des jeunes filles et des femmes à ces messages, et serait liée à des impacts négatifs sur les plans de l'estime de soi, de la dépression, des troubles alimentaires et de la sexualisation précoce (p. ex., Grabe et al., 2007, 2008). Les garçons et les hommes, ciblés par des stéréotypes idéalisant la silhouette mésomorphe, sont aussi à risque de développer des symptômes dépressifs, une faible estime de soi et une image corporelle négative (Hargreaves et Tiggemann, 2009 ; Hausenblas et al., 2013). Si des effets délétères de l'adhésion aux contenus sexualisés ont été documentés chez les adolescents, peu de travaux ont porté sur la situation des jeunes adultes.

\section{Sursexualisation des jeunes adultes}

Chez les jeunes adultes (18-30 ans), l'exploration de l'intimité sexuelle et amoureuse, la formation de relations de couple stables et la consolidation de l'identité constituent des tâches développementales centrales (Arnett, 2004 ; Erikson, 1993). À cet âge, l'adhésion marquée aux contenus sexualisés s'avère alors mieux caractérisée par le terme "sursexualisation ", le préfixe "hyper " décrivant plutôt la sexualisation excessive des mineurs (Brassard et al., 2016). Une série de quatre études menées par Brassard et ses collègues a permis de fournir une définition opérationnelle et de développer un instrument de mesure intégrateur du concept de sursexualisation adulte. Après une première étude qualitative explorant la réalité subjective des jeunes adultes québécois et d'intervenants œuvrant auprès de cette population, Brassard et ses collègues ont défini la sursexualisation comme :

le fait d'adhérer au système de croyances véhiculé par la société qui se traduit par l'utilisation de son corps comme un objet dans le but de plaire, et ce, en sexualisant ses relations à autrui via des comportements de séduction, un discours sexualisé et un large répertoire de pratiques sexuelles. 
Une seconde étude a permis de créer et valider le Questionnaire de sursexualisation adulte (QSA : Brassard et al., 2016). Le QSA mesure six dimensions de la sursexualisation : 1) le surinvestissement de l'apparence sexualisée (intériorisation des normes sexualisées et efforts pour y correspondre) ; 2) l'objectification sexuelle (instrumentalisation de son corps pour plaire) ; 3) le discours sexualisé (ouverture à divulguer les détails intimes de sa sexualité) ; 4) la sexualité axée sur la performance (sexualité calquée sur la pornographie et visant la performance) ;5) l'attitude séductrice (relations régies par le désir de charmer) ; et 6) le sens intime accordé à la sexualité (échelle inversée ; perception de la sexualité comme empreinte de respect, de sens et d'intimité relationnelle, au contraire de la sexualité sans engagement) (Brassard et al., 2016).

Deux études de validation supplémentaires ont permis à Brassard et ses collègues (2016) de confirmer la structure factorielle du QSA et d'appuyer sa validité convergente. La plupart des dimensions de la sursexualisation sont liées à des corrélats négatifs, tels qu'une faible estime de soi, une perception négative de son corps, de l'anxiété à l'égard de la sexualité, de la détresse associée à son vécu sexuel, des rapports sexuels survenant plus tôt ainsi que des comportements sexuels à risque d'infections transmissibles sexuellement. Or, il est apparu que le discours sexualisé était plutôt lié à des aspects positifs chez les jeunes adultes, comme l'estime de soi sexuelle et l'affirmation de soi sexuelle, tout en étant associé à la prise de risques sexuels. Le sens intime accordé à la sexualité, inverse de la sursexualisation, s'est avéré lié à des aspects positifs du bien-être individuel (Brassard et al., 2016). Ces conclusions font penser que la sursexualisation est un construit non pathologique reflétant à quel point l'individu intègre les messages culturels sexualisés. II existe toutefois un important manque de connaissances sur les caractéristiques individuelles des jeunes adultes qui ont intégré ces messages dans la construction de leur sexualité. On ignore également si ces jeunes adultes présentent des difficultés particulières dans leur fonctionnement sexuel. Documenter leur profil sociodémographique pourrait permettre de cibler les jeunes adultes particulièrement à risque d'adopter des conduites et attitudes sursexualisées. Ces derniers pourraient bénéficier de modalités de sensibilisation visant à leur permettre de porter un regard critique sur leur sexualité, afin de favoriser une sexualité respectueuse et satisfaisante.

\section{Fonctionnement sexuel}

Bien que la prévalence des difficultés sexuelles soit plus élevée avec l'âge, une étude épidémiologique américaine révèle que $32 \%$ des femmes de 18 à 29 ans rapportent un faible désir sexuel, $19 \%$ des difficultés de lubrification, $21 \%$ de la douleur sexuelle ou des difficultés à atteindre l'orgasme (Laumann, Paik et Rosen, 1999). Pour ce qui est des hommes de ce groupe d'âge, $14 \%$ rapportent un faible désir, $7 \%$ des difficultés érectiles et $37 \%$ des difficultés à contrôler ou atteindre l'orgasme. II apparait donc très pertinent d'explorer si les conduites de sursexualisation des jeunes adultes peuvent être associées à ces difficultés. La présente étude se penche sur six difficultés possibles sur le plan de la réponse sexuelle, soit les problèmes concernant le désir, l'excitation, l'érection/la lubrification vaginale, la capacité à atteindre l'orgasme, la satisfaction tirée de l'orgasme ainsi que la douleur sexuelle.

Fonctionnement sexuel et conduites sursexualisées. Bien qu'aucun chercheur n'ait examiné spécifiquement le concept de sursexualisation, des études ont montré que l'objectification de soi et les préoccupations quant à l'apparence, un concept connexe au surinvestissement de l'apparence, sont reliées aux difficultés sexuelles chez les femmes (Nobre et Pinto-Gouveia, 2008 ; Steer et Tiggemann, 2008) et chez les hommes (Aubrey, 2007 ; Sanchez et Kiefer, 2007). II semble donc possible que le 
surinvestissement de l'apparence et l'objectification sexuelle soient associés à des difficultés sexuelles chez les jeunes adultes.

À titre de concept connexe à la sexualité axée sur la performance, des études ont examiné le rôle de l'anxiété de performance sur la fonction sexuelle. Leurs résultats sont contradictoires, montrant à la fois les effets inhibants et stimulants de l'anxiété de performance sur l'excitation sexuelle (Van den Hout et Barlow, 2000). Les études ayant plutôt porté sur le visionnement de pornographie révèlent pour leur part l'absence de lien (Landripet et Stulhofer, 2015) ou la présence de liens négatifs entre celui-ci et les difficultés sexuelles chez les adultes (Blais-Lecours et al., 2016). II ne semble donc pas possible de se prononcer sur les liens envisagés entre la sexualité axée sur la performance et la fonction sexuelle.

Dans la documentation scientifique, le concept s'apparentant le plus au discours sexualisé semble être la communication sexuelle, puisque ces deux concepts incluent une absence de tabous sexuels ainsi qu'une ouverture et une aisance à aborder ce sujet. De fait, un lien positif entre le discours sexualisé et l'affirmation sexuelle est documenté par Brassard et al. (2016), et plusieurs écrits révèlent que le fait de communiquer avec un partenaire sur le plan sexuel est relié à une moindre présence de dysfonctions sexuelles (p. ex., Hayes et al., 2008 ; Rehman, Rellini et Fallis, 2011). Compte tenu des liens théoriques et empiriques entre communication sexuelle et discours sexualisé, un lien négatif serait attendu entre le discours sexualisé et les difficultés sexuelles.

Enfin, la dimension du sens intime accordé à la sexualité est également un nouveau concept n'ayant pas fait l'objet d'écrits scientifiques à ce jour. Des études ont montré une plus faible prépondérance de difficultés sexuelles chez les couples engagés (p. ex., Pederson et Blekesaune, 2003 ; Richters et al., 2006), et ce, surtout pour les femmes (p. ex., Mah et Binik, 2001). De la même manière, aucune étude ne s'est intéressée aux liens entre l'adoption d'une attitude et de comportements de séduction dans les relations interpersonnelles et la présence de difficultés sexuelles. Ces constats incitent à poursuivre la recherche en matière de sexualité des jeunes adultes et, en particulier, à explorer le rôle de la sursexualisation. 


\section{Objectifs}

Le premier objectif de la présente étude vise à explorer les liens entre chacune des dimensions de la sursexualisation adulte (surinvestissement de l'apparence, objectification, discours sexualisé, séduction, sexualité axée sur la performance, faible sens intime accordé à la sexualité) et les caractéristiques sociodémographiques des jeunes adultes. Puisqu'aucune étude publiée ne permet de formuler d'hypothèses, les liens entre les dimensions de la sursexualisation et les caractéristiques sociodémographiques des jeunes adultes sont abordés de manière exploratoire (Question de recherche 1). Le second objectif vise à vérifier les liens entre les conduites sursexualisées et les difficultés sexuelles des jeunes adultes. II est attendu que l'objectification de soi et le surinvestissement de l'apparence soient liés positivement à la présence de difficultés sexuelles (Hypothèse 1), mais que le discours sexualisé y soit lié négativement (Hypothèse 2). Les liens entre les autres dimensions de la sursexualisation et les difficultés sexuelles seront étudiés de manière exploratoire (Question de recherche 2).

\section{MÉTHODE}

\section{Participants et procédure}

Un échantillon de 1275 jeunes adultes canadiens francophones âgés de 18 à 30 ans $(M=21,31$; $E ́ T=3,18$ ) et sexuellement actifs a été recruté par le biais de courriels transmis aux étudiants universitaires et de publicités sur les réseaux sociaux. Parmi eux, 982 sont des femmes (77,0 \%) et 293 sont des hommes $(23,0 \%)$. Ils sont principalement étudiants $(72,4 \%)$, les autres sont travailleurs $(25,6 \%)$ ou les deux $(2,0 \%)$. Les participants ont en moyenne 14 années de scolarité $(E ́ T=2,55)$ et un revenu annuel moyen de $16753 \$(E ́ T=34859)$. Ils sont pour la plupart en relation de couple exclusive $(44,7 \%)$, conjoints de fait $(23,0 \%)$ ou mariés $(3,2 \%)$, tandis qu'une moindre proportion sont célibataires $(24,0 \%)$ ou ont des fréquentations non exclusives $(4,9 \%)$. Les adultes en couple sont majoritairement en relation de couple hétérosexuelle (91,7\%), et ce, depuis en moyenne 2,26 ans $(E ́ T=2,21)$. Les participants volontaires ont répondu de façon anonyme à une série de questionnaires en ligne sur un site sécurisé (durée : 30 minutes). Le projet de recherche a été approuvé par le comité d'éthique de l'institution des chercheurs.

\section{Instruments}

Un questionnaire sociodémographique a permis d'amasser des informations sur le genre des participants, leur âge, leur nombre d'années de scolarité, leur occupation principale, leur revenu, leur statut conjugal et, s'il y a lieu, la durée de leur relation de couple et le genre du partenaire. Une question a porté sur l'existence d'une expérience d'agression sexuelle dans l'enfance (oui ou non).

Le Questionnaire de sursexualisation adulte (QSA ; Brassard et al., 2016) évalue les conduites et attitudes sursexualisées au moyen de 30 items, sur une échelle de Likert allant de (1) fortement en désaccord à (6) fortement en accord. Les scores des six sous-échelles sont formés de la moyenne des items correspondant aux dimensions du surinvestissement de l'apparence $(\alpha=0,83)$, de l'objectification sexuelle $(\alpha=0,84)$, du discours sexualisé $(\alpha=0,85)$, de l'attitude séductrice $(\alpha=0,83)$, de la sexualité axée sur la performance $(\alpha=0,69)$ et du sens intime accordé à la sexualité (échelle inversée ; $\alpha=0,66$ ). Les qualités psychométriques du QSA (fidélité, structure factorielle, validité convergente) sont établies 
auprès de trois échantillons de jeunes adultes (Brassard et al., 2016).

Le Arizona Sexual Experiences Scale (ASEX; McGahuey et al., 2000) évalue la présence de cinq difficultés sexuelles chez les adultes : désir, excitation, érection/lubrification, capacité à atteindre l'orgasme et satisfaction tirée de l'orgasme. Chaque item est évalué sur une échelle Likert variant de (1) hyperfonction à (6) hypofonction. Sa cohérence interne $(\alpha=0,91)$ et sa fidélité test-retest à deux semaines d'intervalle (patients : $r=0,80$; contrôles : $r=0,89$ ) se sont montrées adéquates lors de sa validation (McGahuey et al., 2000). La version française du ASEX de Brassard et Bourassa (2012) possède un sixième item évaluant la douleur sexuelle. La cohérence interne des six items est adéquate dans le présent échantillon $(\alpha=0,73)$.

\section{RÉSULTATS}

Pour vérifier le premier objectif de recherche, quatre analyses de variance multivariées (MANOVA) ont été menées à l'aide du logiciel SPSS 22 sur les scores des six sous-échelles en fonction des variables sociodémographiques catégorielles suivantes : genre, genre du partenaire, statut conjugal, agression sexuelle dans l'enfance (ASE). Les postulats des analyses (normalité, homogénéité des variances et covariances) ont été vérifiés. Les résultats révèlent que les femmes présentent des scores plus élevés de surinvestissement de l'apparence et de sens intime accordé à la sexualité, alors que les hommes adoptent davantage une sexualité axée sur la performance (voir le tableau 1). De plus, les participants en couple avec un partenaire de même genre rapportent des scores plus élevés de discours sexualisé, d'attitude séductrice et de sexualité axée sur la performance.

Tableau 1. Moyennes des scores de sursexualisation selon les variables sociodémographiques

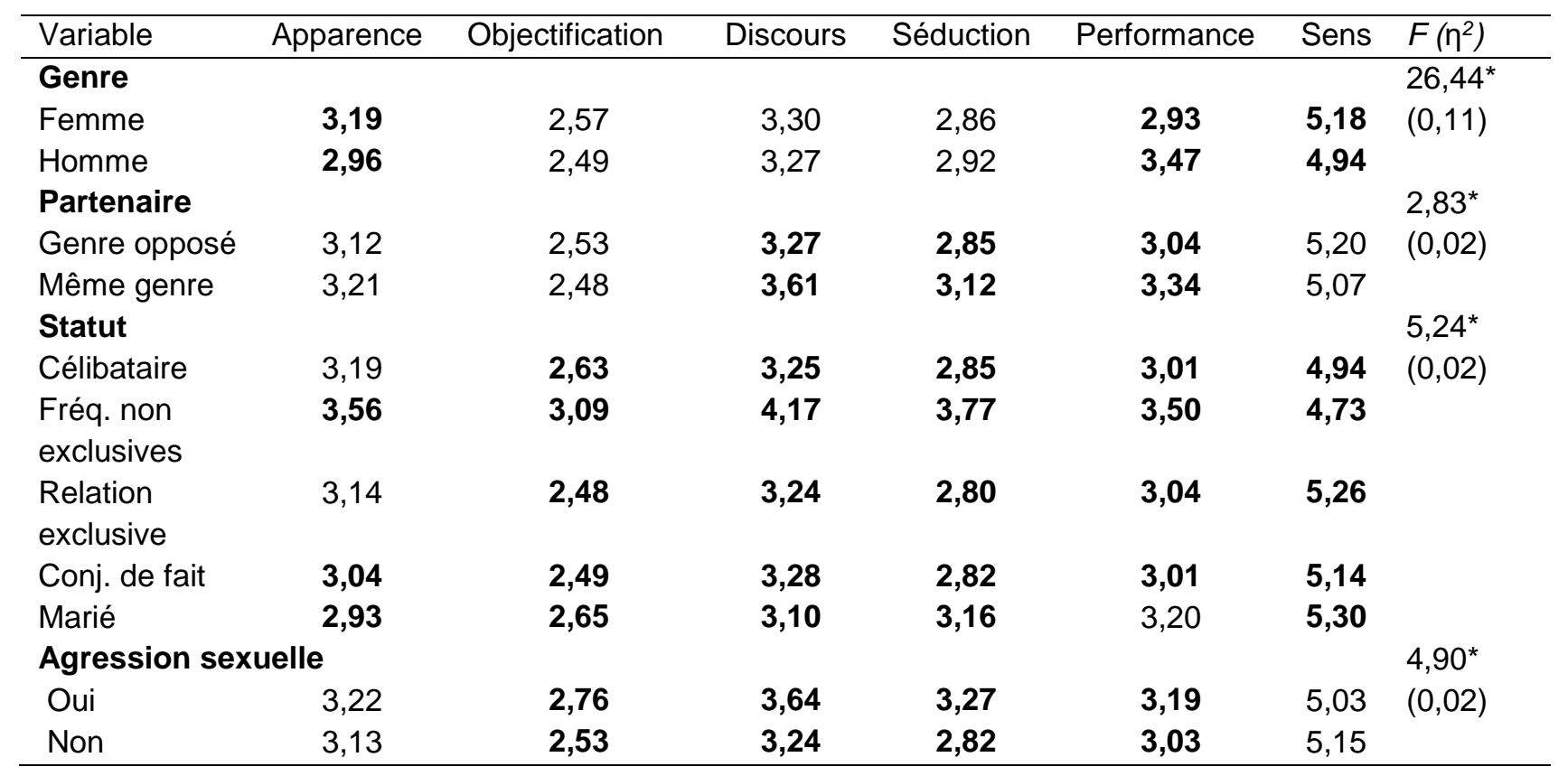

${ }^{*} p<0,05$. Note. Les moyennes en gras sont significativement différentes au seuil de $p<0,05$ dans les analyses de variance univariées menées à la suite des analyses multivariées significatives.

En ce qui concerne le statut conjugal, les comparaisons multiples de Tukey révèlent que les adultes en 
situation de fréquentations non exclusives présentent des scores plus élevés de surinvestissement de l'apparence que les adultes mariés ou conjoints de fait. Ils rapportent aussi davantage d'objectification, de discours sexualisé et de séduction que tous les autres statuts, mais ils ne diffèrent pas des adultes mariés quant à la sexualité axée sur la performance. Les adultes célibataires et ceux qui ont des fréquentations non exclusives rapportent un plus faible sens intime accordé à la sexualité que les adultes en relation de couple exclusive, conjoints de fait ou mariés. Enfin, les participants qui disent avoir vécu une agression sexuelle dans l'enfance montrent des scores plus élevés d'objectification, de discours sexualisé, de séduction et de sexualité axée sur la performance que ceux qui disent ne pas avoir vécu d'ASE.

Des analyses de corrélation de Pearson ont ensuite été menées entre les scores aux échelles de sursexualisation et les variables sociodémographiques continues (âge, nombre d'années de scolarité, revenu, durée de la relation). Les résultats révèlent que plus l'âge est élevé, plus le surinvestissement de l'apparence est faible $(r=-0,09, p=0,002)$. Un nombre d'années de scolarité plus élevé est relié à des scores plus faibles de surinvestissement de l'apparence $(r=-0,10, p<0,001)$, de discours sexualisé $(r=-0,07, p=0,011)$ et de sexualité axée sur la performance $(r=-0,08, p=0,004)$, ainsi qu'à un degré plus élevé de sens intime accordé à la sexualité $(r=0,08, p=0,006)$. Un revenu plus élevé est lié positivement à la sexualité axée sur la performance $(r=0,07, p=0,019)$. Enfin, chez les adultes en couple, la durée de la relation est liée à des scores plus faibles de surinvestissement de l'apparence $(r=-0,08, p=0,011)$, de séduction $(r=-0,07, p=0,035)$ et de sexualité axée sur la performance $(r=-0,10, p=0,002)$.

Pour répondre au deuxième objectif de recherche, six analyses de régression multiple hiérarchique ont été menées à l'aide du logiciel SPSS 22 sur les difficultés sexuelles, en entrant les variables démographiques (genre, âge, scolarité, revenu) à l'étape 1 et les six sous-échelles de sursexualisation à l'étape 2. Pour tenir compte du nombre d'analyses et prévenir l'inflation de l'erreur de type 1, un seuil alpha de 0,008 (correction de Bonferroni ; 0,05/6) a été retenu pour juger de la signification d'une variable prédictive. Les postulats de la régression ont été vérifiés (normalité des résidus, absence de multicolinéarité, valeurs extrêmes multivariées).

Les résultats présentés au tableau 2 révèlent que les femmes présentent davantage de difficultés sexuelles que les hommes, et ce, pour toutes les difficultés. Par ailleurs, le discours sexualisé est relié à moins de difficultés sexuelles, soit à une meilleure libido, une meilleure excitation, une meilleure capacité d'érection/lubrification, ainsi qu'à une meilleure capacité orgasmique et une plus grande satisfaction tirée de l'orgasme. La sexualité axée sur la performance est liée à un meilleur désir et une plus grande excitation sexuelle. Toutefois, l'objectification sexuelle est reliée à une plus faible satisfaction envers l'orgasme, alors que le sens intime accordé à la sexualité est lié à une meilleure satisfaction tirée de l'orgasme. 
Tableau 2. Régressions multiples hiérarchiques reliant les scores de sursexualisation aux difficultés sexuelles, en contrôlant pour les caractéristiques sociodémographiques

\begin{tabular}{|c|c|c|c|c|c|c|}
\hline Variable & Désir & Excitation & $\begin{array}{l}\text { Érection / } \\
\text { lubrification }\end{array}$ & Orgasme & $\begin{array}{l}\text { Satisfaction } \\
\text { orgasme }\end{array}$ & Douleur \\
\hline \multicolumn{7}{|l|}{ Étape 1} \\
\hline $\begin{array}{l}\text { Genre } \\
\text { (réf = homme) }\end{array}$ & $0,14^{\star *}$ & $0,16^{\star *}$ & $0,22^{* *}$ & $0,29^{* *}$ & $0,11^{*}$ & $0,30^{* *}$ \\
\hline Âge & 0,08 & 0,01 & 0,02 & $-0,01$ & $-0,03$ & 0,02 \\
\hline Scolarité & 0,07 & 0,07 & 0,07 & $-0,03$ & $-0,04$ & 0,02 \\
\hline Revenu & $-0,02$ & $-0,01$ & $-0,04$ & $-0,05$ & 0,01 & $-0,08$ \\
\hline \multicolumn{7}{|l|}{ Étape 2} \\
\hline Apparence & 0,06 & 0,03 & 0,07 & 0,01 & 0,04 & 0,10 \\
\hline Objectification & $-0,03$ & $-0,04$ & $-0,01$ & 0,09 & $0,15^{*}$ & $-0,01$ \\
\hline Discours & $-0,24^{* *}$ & $-0,26^{* *}$ & $-0,14^{\star *}$ & $-0,19^{\star *}$ & $-0,14^{* *}$ & $-0,05$ \\
\hline Séduction & $-0,06$ & 0,03 & $-0,02$ & $-0,01$ & $-0,10$ & $-0,02$ \\
\hline Performance & $-0,22^{\star *}$ & $-0,17^{\star *}$ & $-0,07$ & $-0,02$ & $-0,04$ & $-0,08$ \\
\hline Sens & $-0,08$ & $-0,07$ & $-0,05$ & $-0,09$ & $-0,16^{\star *}$ & $-0,01$ \\
\hline$R^{2}{ }_{a j}$ & 0,20 & 0,15 & 0,08 & 0,13 & 0,06 & 0,12 \\
\hline
\end{tabular}

${ }^{*} p<0,008{ }^{* *} p<0,001$

\section{DISCUSSION}

La présente étude avait pour but d'explorer le profil sociodémographique et sexuel des jeunes adultes adoptant des conduites sursexualisées. Les résultats concernant le premier objectif ont révélé que le genre, le genre du partenaire, le statut conjugal, le niveau de scolarité ainsi que l'existence d'une expérience d'agression sexuelle dans l'enfance constituent des facteurs permettant de distinguer les jeunes adultes quant à leur degré d'adoption des attitudes et conduites sursexualisées.

Genre. Un premier constat en ce qui a trait aux différences liées au genre est que les jeunes femmes adultes rapportent davantage de surinvestissement de l'apparence sexualisée et de sens intime accordé à la sexualité que les hommes. Ce résultat pourrait s'expliquer par les normes concernant les rôles de genre, selon lesquelles les femmes doivent investir dans leur apparence, rechercher la minceur et valoriser les relations amoureuses exclusives (Mahalik et al., 2005). Bien que les médias ciblent de plus en plus les idéaux d'image corporelle des hommes (Ricciardelli et Williams, 2012), ceux-ci sont davantage socialisés à ne pas rechercher l'approbation des autres (self-reliance) et à voir la sexualité sans engagement comme une source de valorisation (Mahalik et al., 2003). Les hommes de la présente étude ont aussi, par rapport aux femmes, davantage rapporté une sexualité axée sur la performance. Puisque cette dimension reflète les conduites sexuelles calquées sur les standards pornographiques et centrées sur la performance sexuelle, ce résultat pourrait s'expliquer, notamment, par la plus grande tendance des hommes à consommer de la pornographie (Blais-Lecours et al., 2016 ; Wright et Bae, 2015).

Une seconde observation en rapport avec le genre est que les adultes en couple avec un partenaire de même genre ont rapporté davantage de discours sexualisé, d'attitude séductrice et de sexualité axée sur la performance que les adultes en relation avec un partenaire de genre opposé. Ce résultat pourrait 
refléter une relation à l'autre généralement plus sexualisée chez les adultes en couple de même genre, qui s'exprimerait à la fois verbalement, dans la séduction, ainsi que dans une sexualité influencée par la pornographie. De fait, la consommation de pornographie est plus fréquente chez les hommes en relation avec un homme (Downing et al., 2017) et les médias destinés à la communauté homosexuelle présenteraient des formes plus prononcées de sexualisation (Duggan et McCreary, 2004). Cependant, une étude comparative n'a pas trouvé de différence sur le plan de la communication sexuelle entre les adultes en relation avec un partenaire de genre opposé et ceux en relation avec un partenaire de même genre, bien que le désir sexuel soit plus élevé chez ces derniers (Holmberg et Blair, 2009).

Statut conjugal. De façon originale, les résultats de cette étude montrent que les adultes ayant des fréquentations non exclusives tendent à adopter toutes les conduites sursexualisées dans une plus grande mesure que les adultes célibataires ou en relation de couple. En effet, ils surinvestissent davantage leur apparence que les adultes mariés ou conjoints de fait. Ils rapportent aussi les scores les plus élevés d'objectification, de discours sexualisé, de séduction et de sexualité axée sur la performance, bien qu'ils ne diffèrent pas des adultes mariés sur ce dernier aspect. Ces résultats font écho aux propositions de Brassard et ses collègues (2016), selon lesquelles le sens intime accordé à la sexualité s'oppose à la sexualité sans engagement en encourageant la réflexion quant au respect de soi et de l'autre, en plus de stimuler le désir de vivre une sexualité intime dans une relation amoureuse stable. II apparait donc que la nature même des relations dans les fréquentations non exclusives, où le sens intime accordé à la sexualité est nécessairement plus faible, est intimement liée à la sursexualisation des jeunes adultes.

Âge et scolarité. Les résultats ont aussi révélé que l'âge et le nombre d'années de scolarité des répondants sont liés à un moindre surinvestissement de l'apparence, mais que seule la scolarité est liée à une plus faible adoption du discours sexualisé et de la sexualité axée sur la performance et à un plus grand sens intime accordé à la sexualité. Bien qu'aucune étude n'ait examiné cette question, il est possible de postuler que les jeunes adultes qui poursuivent des études plus avancées pourraient développer une meilleure réflexion critique à l'égard des messages sexualisés ou encore avoir intégré d'autres facettes de leur identité au-delà de l'apparence physique, telles que leurs intérêts, leurs compétences ou leurs traits de personnalité.

ASE. L'expérience d'agression sexuelle dans l'enfance s'avère aussi un facteur de risque concernant l'adoption de conduites d'objectification sexuelle, de discours sexualisé, d'attitudes et comportements de séduction et d'une sexualité axée sur la performance. Comme cette expérience touche l'enfant au cœur de ses premières représentations de la sexualité, il n'est pas surprenant que ces expériences sexuelles précoces altèrent le rapport à soi et aux autres en matière de sexualité. Chez les adolescents, l'ASE a en effet été liée à la précocité sexuelle, au nombre élevé de partenaires sexuels, à la sexualité à risque et à la grossesse (Loeb et al., 2002). En concordance avec nos résultats, les études sur l'ASE montrent que celle-ci augmente aussi la probabilité de vivre des difficultés sexuelles et amoureuses à l'âge adulte, dont la sexualité compulsive et l'infidélité (Vaillancourt-Morel et al., 2015 ; 2016).

Difficultés sexuelles. Les résultats concernant le deuxième objectif appuient partiellement les hypothèses formulées et révèlent des liens nuancés entre les conduites de sursexualisation et les difficultés sexuelles des jeunes adultes, en contrôlant pour les caractéristiques sociodémographiques. D'abord, l'objectification sexuelle, mais pas le surinvestissement de l'apparence, est liée à une plus faible satisfaction envers l'orgasme. Il est possible d'expliquer ce résultat en s'appuyant sur la théorie de l'objectification, qui soutient que l'intériorisation des standards de beauté véhiculés dans les médias 
est susceptible de générer de l'anxiété et de la honte envers son corps (Aubrey, 2007 ; Calogero et Thompson, 2009 ; Fredrickson et Roberts, 1997 ; Sanchez et Kiefer, 2007). Ces émotions négatives, jumelées au désir de plaire, amèneraient la personne à ne pas profiter du moment présent pendant les relations sexuelles, ce qui nuirait au plaisir sexuel (Sanchez et Keifer, 2007 ; Steer et Tiggeman, 2008).

Le sens intime accordé à la sexualité est aussi lié à la satisfaction tirée de l'orgasme. Il est possible que l'adoption d'une vision de la sexualité empreinte de respect, de sens et d'intimité dans une relation engagée favorise le plaisir sexuel. Selon Armstrong, England et Fogarty (2012), les personnes engagées dans des unions stables ont davantage tendance à varier leurs pratiques sexuelles, à mieux connaître les préférences de leur partenaire et à exprimer de l'affection, ce qui contribuerait au plaisir et à la fréquence de l'orgasme chez les femmes. Au contraire, dans les relations sans engagement, les hommes se préoccuperaient peu du plaisir de leur partenaire tandis que les femmes prioriseraient le plaisir de l'homme au détriment de leur propre plaisir (Armstrong et al., 2012).

En accord avec les hypothèses, le discours sexualisé s'est avéré lié à moins de difficultés sexuelles, notamment au niveau du désir, de l'excitation, de la capacité d'érection/lubrification, ainsi que de l'atteinte de l'orgasme et de la satisfaction tirée de celui-ci. Cela concorde avec les conclusions de Brassard et ses collègues (2016), où l'adoption d'un discours sexualisé est apparue bénéfique pour le jeune adulte, puisqu'il se manifeste par une ouverture à discuter de la sexualité dans le contexte plus intime des relations sexuelles. Plusieurs études appuient le rôle important de la communication sexuelle dans la prévention et le traitement des dysfonctions sexuelles (p. ex., Hayes et al., 2008 ; Rehman et al., 2011).

De façon surprenante, la sexualité axée sur la performance s'est avérée liée à une meilleure libido (moins de difficultés au niveau du désir) et à une plus grande excitation sexuelle. II est possible que ces relations soient bidirectionnelles. D'un côté, le visionnement de pornographie en couple peut élargir le répertoire sexuel et nourrir le désir et l'excitation des partenaires (Bridges et Morokoff, 2011). D'un autre côté, une forte libido chez le jeune adulte peut expliquer que celui-ci calque ses pratiques sexuelles sur la pornographie qu'il consomme.

\section{Retombées}

La présente étude permet de fournir un premier portrait sociodémographique et sexuel nuancé des jeunes adultes en rapport avec les conduites de sursexualisation. Les constats qui en émergent montrent que ces conduites peuvent avoir des effets négatifs sur la sexualité, mais également, dans certains cas, des effets positifs, et permettent de générer des pistes de prévention et d'intervention pertinentes pour les intervenants œuvrant auprès des jeunes adultes. D'abord, par le biais de conférences et d'ateliers éducatifs, l'intervenant social peut sensibiliser les jeunes adultes aux conduites de sursexualisation en leur permettant de mieux les identifier et les comprendre. II peut ainsi susciter chez ces derniers une réflexion plus critique sur le sujet et sur la sexualité en général, tout en faisant la promotion des valeurs de respect de soi et de l'autre dans les relations intimes et amoureuses. La connaissance et l'évaluation des conduites sursexualisées chez les jeunes adultes, par exemple à l'aide du QSA (Brassard et al., 2016), peuvent aussi permettre aux intervenants en suivi individualisé d'identifier plus clairement certains facteurs de risque et de protection relativement aux difficultés sexuelles vécues par les jeunes adultes. L'intervenant peut par la suite faciliter la reconnaissance et la compréhension de ces facteurs chez les jeunes adultes qui le consultent et leur proposer un plan 
d'intervention approprié pour contrer leurs difficultés sexuelles en considérant leurs conduites sursexualisées. L'intervenant peut, entre autres, encourager et soutenir chez les jeunes adultes en consultation le développement d'un regard critique à l'égard de leur sexualité, des stratégies personnelles pour contrer la pression de vivre des relations sexuelles sans engagement, et de meilleures capacités de communication sexuelle avec leur partenaire (Brassard et al., 2016), le tout dans une optique de bien-être et d'épanouissement sexuel. Encadrés par l'intervenant social, les jeunes adultes peuvent ainsi viser un changement dans leurs conduites et comportements d'ordre intime et sexuel en donnant un sens plus profond à leur sexualité, pour ultimement accéder à une sexualité saine, respectueuse et satisfaisante.

\section{Limites et pistes pour la recherche future}

Bien qu'originale, cette étude corrélationnelle et transversale repose uniquement sur des questionnaires autorapportés. Malgré les efforts pour recruter un échantillon vaste et diversifié, l'homogénéité de l'échantillon en termes de genre, d'orientation et d'occupation limite la généralisation des résultats à l'ensemble des jeunes adultes. Malgré la robustesse des contrôles statistiques, la puissance statistique élevée des analyses mène à des résultats reposant sur de faibles tailles d'effet, ce qui invite à considérer l'ensemble des résultats avec prudence. L'interprétation des liens corrélationnels est aussi limitée par la difficulté que pose l'estimation du statut socioéconomique des jeunes adultes puisque leur scolarité n'est peut-être pas terminée et que leur revenu est susceptible de varier. Des études longitudinales sur des échantillons représentant davantage les minorités sexuelles seraient souhaitables afin de clarifier les relations réciproques existant entre des facteurs de risque et de protection, la sursexualisation et le fonctionnement sexuel au fil du développement psychosexuel des jeunes adultes. Des études dyadiques évaluant les deux partenaires pourraient aussi enrichir la compréhension de la sexualité des jeunes adultes. 


\section{RÉFÉRENCES}

American Psychological Association Task Force on the Sexualization of Girls (2007). Report of the APA Task Force on the Sexualization of Girls, Washington, DC, American Psychological Association. Repéré à www.apa.org/pi/wpo/ sexualization.html

Armstrong, E. A., England, P. et Fogarty, A. (2012). « Accounting for women's orgasm and sexual enjoyment in college hookups and relationships », American Sociological Review, vol. 77, $n^{\circ} 3$, p. 435-462.

Arnett, J. J. (2004). Emerging adulthood: The winding road from the late teens through the twenties, New York, Oxford University Press.

Aubrey, J. S. (2007). "The impact of sexually objectifying media exposure on negative body emotions and sexual self-perceptions: Investigating the mediating role of body self-consciousness », Mass Communication, vol. 10, $\mathrm{n}^{\circ} 1$, p. 1-23.

Blais, M., Raymond, S., Manseau, H. et Otis, J. (2009). « La sexualité des jeunes Québécois et Canadiens. Regard critique sur le concept d'“hypersexualisation” », Globe, vol. 12, n², p. 23-46.

Blais-Lecours, S., Vaillancourt-Morel, M.-P., Sabourin, S. et Godbout, N. (2016). « Cyberpornography: Time use, perceived addiction, sexual functioning, and sexual satisfaction », Cyberpsychology, Behavior, and Social Networking, vol. 19, n 11, p. 649-655.

Brassard, A. et Bourassa, M. (2012). Traduction française du Arizona Sexual Experience Scale (ASEX), document inédit, Sherbrooke, Université de Sherbrooke.

Brassard, A., Houde, F., Caouette, C., Lussier, Y. et de Pierrepont, C. (2016). Validation of a new questionnaire assessing adult oversexualization, manuscrit soumis pour publication.

Bridges, A. J. et Morokoff, P. J. (2011). « Sexual media use and relational satisfaction in heterosexual couples », Personal Relationships, vol. 18, n 4, p. 562-585.

Calogero R. M. et Thompson, J. K. (2009). « Potential implications of the objectification of women's bodies for women's sexual satisfaction », Body Image, vol. 6, n² 2, p. 145-148.

Downing, M. J., Schrimshaw, E. W., Scheinmann, R., Antebi-Gruszka, N. et Hirshfield, S. (2017). "Sexually explicit media use by sexual identity: A comparative analysis of gay, bisexual, and heterosexual men in the United States », Archives of Sexual Behavior, vol. 46, n 6, p. 1763-1776.

Duggan, S. J. et McCreary, D. R. (2004). « Body image, eating disorders, and the drive for muscularity in gay and heterosexual men », Journal of Homosexuality, vol. 47, nos 3-4, p. 45-58.

Duquet, F. et Quéniart, A. (2009). Perceptions et pratiques de jeunes du secondaire face à l'hypersexualisation et à la sexualisation précoce, rapport de recherche issu du projet «Outiller les jeunes face à l'hypersexualisation », Université du Québec à Montréal. Repéré à 
https://www.csestuaire.qc.ca/sites/default/files/Rapport_recherche.pdf

Erikson, E. H. (1993). Identity and the life cycle, New York, Norton.

Fredrickson, B. L. et Roberts, T. (1997). « Objectification theory: Toward understanding women's lived experiences and mental health risks », Psychology of Women Quarterly, vol. 21, n² 2, p. 173-206.

Gerbner, G., Gross, L., Morgan, M. et Signorielli, N. (1994). «Growing up with television: The cultivation perspective », dans J. Bryant et D. Zillmann (dir.), Media effects: Advances in theory and research, Hillsdale, NJ, Lawrence Erlbaum, p. 17-41.

Grabe, S., Hyde, J. S. et Lindberg, S. M. (2007). « Body objectification and depression in adolescents: The role of gender, shame, and rumination », Psychology of Women Quarterly, vol. 31, $\mathrm{n}^{\circ} 2$, p. 164-175.

Grabe, S., Ward, L. M. et Hyde, J. S. (2008). " The role of the media in body image concerns among women: A meta-analysis of experimental and correlational studies », Psychological Bulletin, vol. 134, n³, p. 460-476.

Hargreaves, D. A. et Tiggemann, M. (2009). « Muscular ideal media images and men's body image: Social comparison processing and individual vulnerability », Psychology of Men \& Masculinity, vol. $10, n^{\circ} 2$, p. 109-119.

Hausenblas, H., Campbell, A., Menzel, J. E., Doughty, J., Levine, M. et Thompson, J. K. (2013). «Media effects of experimental presentation of the ideal physique on eating disorder symptoms: A meta-analysis », Clinical Psychology Review, vol. 33, n 1, p. 168-181.

Hayes, R. D., Dennerstein, L., Bennett, C. M., Sidat, M., Gurrin, L. C. et Fairley, C. K. (2008). « Risk factors for female sexual dysfunction in the general population: Exploring factors associated with low sexual function and sexual distress », International Society for Sexual Medicine, vol. 5, $\mathrm{n}^{\circ}$ 7, p. 1681-1693.

Holmberg, D. et Blair, K. L. (2009). « Sexual desire, communication, satisfaction, and preferences of men and women in same-sex versus mixed-sex relationships », Journal of Sex Research, vol. 46, $\mathrm{n}^{\circ} 1$, p. $57-66$.

Lambert, G., Mathieu-Chartier, S., Goggin, P., Maurais, E. et les membres de l'équipe PIXEL (2017). Étude PIXEL-Portrait de la santé sexuelle des jeunes adultes québécois, Québec, Institut national de santé publique du Québec. Repéré à

https://www.inspq.qc.ca/sites/default/files/publications/2307_pixel_portrait_sante_sexuelle_jeunes_ adultes_quebec.pdf

Landripet, I. et Stulhofer, A. (2015). « Is pornography use associated with sexual difficulties and dysfunctions among younger heterosexual men? ", The Journal of Sexual Medicine, vol. 12, $n^{\circ} 5$, p. 1136-1139. 
Laumann, E. O., Paik, A. et Rosen, R. C. (1999). «Sexual dysfunction in the United States:

Prevalence and predictors », JAMA, vol. 281, n 6, p. 537-544.

Loeb, T. B., Williams, J. K., Carmona, J. V., Rivkin, I., Wyatt, G. E., Chin, D. et Asuan-O'Brien, A. (2002). "Child sexual abuse: Associations with the sexual functioning of adolescents and adults ", Annual Review of Sex Research, vol. 13, p. 307-345.

Mah, K. et Binik, Y. M. (2001). «The nature of human orgasm: A critical review of major trends », Clinical Psychology Review, vol. 21, n 6, p. 823-856.

Mahalik, J. R., Mooray, E. B., Coonerty-Femiano, A., Ludlow, L. H., Slaterry, S. M. et Smiler, A. (2005). «Development of the Conformity to Feminine Norms Inventory », Sex Roles, vol. 52, nºs 7-8, p. 417435.

Mahalik, J. R., Locke, B. D., Ludlow, L. H., Diemer, M. A., Scott, R. P. J., Gottfried, M. et Freitas, G. (2003). «Development of the Conformity to Masculine Norms Inventory », Psychology of Men \& Masculinity, vol. 4, $\mathrm{n}^{\circ} 1$, p. 3-25.

McGahuey, C. A., Gelenberg, A. J., Laukes, C. A., Moreno, F. A., Delgado, P. L., McKnight, K. M. et Manber, R. (2000). «The Arizona Sexual Experiences Scale (ASEX): Reliability and validity », Journal of Sex and Marital Therapy, vol. 26, n 1, p. 25-40.

Nalkur, P. G., Jamieson, P. E. et Romer, D. (2010). «The effectiveness of the Motion Picture Association of America's rating system in screening explicit violence and sex in top-ranked movies from 1950 to 2006 », Journal of Adolescent Health, vol. 47, n 5, p. 440-447.

Nobre, P. J. et Pinto-Gouveia, J. (2008). «Cognitive and emotional predictors of female sexual dysfunctions: Preliminary findings », Journal of Sex and Marital Therapy, vol. 34, n 4, p. 325-342.

Pederson, W. et Blekesaune, M. (2003). "Sexual satisfaction in young adulthood: Cohabitation, committed dating or unattached life? », Acta Sociologica, vol. 46, n 3, p. 179-193.

Poulin, R. (2008). Enfances dévastées, tome 2 : Pornographie et hypersexualisation, Ottawa, L'Interligne.

Rehman, U. S., Rellini, A. H. et Fallis, E. (2011). "The importance of sexual self-disclosure to sexual satisfaction and functioning in committed relationships », Journal of Sexual Medicine, vol. 8, $n^{\circ} 11$, p. 3108-3115.

Ricciardelli, L. A. et Williams, R. J. (2012). «Beauty over the centuries - Male », dans T. F. Cash (dir.), Encyclopedia of body image and human appearance. San Diego, CA, Elsevier Academic Press, p. 50-57.

Richard-Bessette, S. (2006). Lexique sur les différences sexuelles, le féminisme et la sexualité, document inédit, Montréal, Université du Québec à Montréal.

Richters, J., Visser, R., Rissel, C. et Smith, A. (2006). « Sexual practices in last heterosexual encounter 
and occurrence of orgasm in a national survey », Journal of Sex Research, vol. 43, n 3, p. 217-226.

Sanchez, D. T. et Kiefer, A. K. (2007). "Body concerns in and out of the bedroom: Implications for sexual pleasure and problems », Archives of Sexual Behavior, vol. 36, n³, p. 808-820.

Steer, A. et Tiggemann, M. (2008). "The role of self-objectification in women's sexual functioning », Journal of Social and Clinical Psychology, vol. 27, n 3, p. 205-225.

Vaillancourt-Morel, M.-P., Godbout, N., Labadie, C., Runtz, M., Lussier, Y. et Sabourin, S. (2015). «Avoidant and compulsive sexual behaviors in male and female survivors of childhood sexual abuse », Child Abuse \& Neglect, vol. 40, p. 48-59.

Vaillancourt-Morel, M.-P., Dugal, C., Poirier Stewart, R., Godbout, N., Sabourin, S., Lussier, Y. et Briere, J. (2016). « Extradyadic sexual involvement and sexual compulsivity in male and female sexual abuse survivors », Journal of Sex Research, vol. 53, nos 4-5, p. 614-625.

Van den Hout, M. et Barlow, D. (2000). "Attention, arousal and expectancies in anxiety and sexual disorders », Journal of Affective Disorders, vol. 61, n 3, p. 241-256.

Wright, P. J. (2009). «Sexual socialization messages in mainstream entertainment mass media: A review and synthesis », Sexuality \& Culture: An Interdisciplinary Quarterly, vol. 13, p. 181-200.

Wright, P. J. et Bae, S. (2015). " A national prospective study of pornography consumption and gendered attitudes toward women ", Sexuality \& Culture, vol. 19, n 3, p. 444-463. 